Astronomy Letters, 2016, Vol. 42, No. 8, pp. 544-554.

\title{
The System of Molecular Clouds in the Gould Belt
}

\author{
V.V. Bobylev \\ Pulkovo Astronomical Observatory, St. Petersburg, Russia
}

\begin{abstract}
Based on high-latitude molecular clouds with highly accurate distance estimates taken from the literature, we have redetermined the parameters of their spatial orientation. This system can be approximated by a $350 \times 235 \times 140$ pc ellipsoid inclined by the angle $i=17 \pm 2^{\circ}$ to the Galactic plane with the longitude of the ascending node $l_{\Omega}=337 \pm 1^{\circ}$. Based on the radial velocities of the clouds, we have found their group velocity relative to the Sun to be $\left(u_{0}, v_{0}, w_{0}\right)=(10.6,18.2,6.8) \pm(0.9,1.7,1.5) \mathrm{km}$ $\mathrm{s}^{-1}$. The trajectory of the center of the molecular cloud system in the past in a time interval of $\sim 60$ Myr has been constructed. Using data on masers associated with lowmass protostars, we have calculated the space velocities of the molecular complexes in Orion, Taurus, Perseus, and Ophiuchus. Their motion in the past is shown to be not random.
\end{abstract}

\section{INTRODUCTION}

A giant stellar-gas complex known as the Gould Belt is located near the Sun (Frogel and Stothers 1977; Efremov 1989; Pöppel 1997, 2001; Torra et al. 2000; Olano 2001). A giant cloud of neutral hydrogen called the Lindblad ring (Lindblad 1967, 2000) is associated with it; the system of nearby OB associations (de Zeeuw et al. 1999), open star clusters (Piskunov et al. 2006; Bobylev 2006), and complexes of nearby molecular clouds (Dame et al. 1987; Perrot and Grenier 2003) are its constituents.

The interest in the Gould Belt stems from the fact that it is the region of active star formation nearest to the Sun. The star formation in Orion, Taurus, or Scorpio-Centaurus either has ended quite recently, as in Scorpio-Centaurus (Preibish and Zinnecker, 1999), or is still continuing, as in the $\rho$ Ophiuchi molecular cloud (Palla and Staler 2000; Lada 2015). Several projects aimed at studying the structure and evolution of the Gould Belt and its components are currently being carried out. These include, for example, the photometric "Herschel Gould Belt survey" (Konyves et al. 2015) and the "Gould Belt Very Large Array survey" devoted to the search for young stars in the Gould Belt (Dzib et al. 2015). The list is long, because the surveys are being done in various spectral ranges. Of interest is the astrometric VLBI survey aimed at determining the trigonometric parallaxes and proper motions of radio stars in the Gould Belt (Rivera et al. 2015). For a number of stars, the error in the distances determined by this method is $5 \%$ (Loinard et al. 2008).

The kinematics of the Gould Belt as a whole is quite contradictory (Palouš and Ehlerová 2014; Bobylev 2014). Either expansion or proper rotation (apart from the Galactic rotation) or both effects together are found in its motion (Lindblad 2000; Bobylev 
2006). Precisely what is responsible for the formation of the Gould Belt remains a puzzle. The hypothesis about expansion after a hypernova explosion proposed by Blaauw (1952) was among the first ones. As the simulations by Palouš (2001) showed, this hypothesis runs into such difficulties as the absence of a stellar age gradient along the radius predicted by it or the impossibility to explain the inclination to the Galactic plane. Nevertheless, a number of important results were obtained by Olano (1982), Moreno et al. (1999), Pöppel and Marronetti (2000), and Perrot and Grenier (2003) based on the supernova explosion model.

Lepiné and Duvert (1994) suggested that the nearby complexes of molecular clouds located sufficiently far from the Galactic plane could be formed by the collisions of highvelocity clouds with the Galactic disk. Since each complex was considered individually, the Gould Belt within this model could arise only by chance.

Comerón and Torra $(1992,1994)$ considered the more complex model of an oblique impact of a high-velocity cloud on the Galactic plane. They showed that a structure similar to the Lindblad ring but with a considerably larger size is formed as a result. It is interesting to note the model by Bekki (2009), which is similar to the Comerón-Torra model, but a cloud of dark matter appears here instead of the high-velocity hydrogen cloud. Bekki's numerical simulations showed that the Gould Belt could be formed 30 Myr ago from an initial gas cloud with a mass of $\sim 10^{6} M_{\odot}$ after its collision with a dark matter clump with a mass of $3 \times 10^{7} M_{\odot}$.

The goal of this paper is to refine the spatial orientation parameters of the system of nearby high-latitude molecular clouds with known distance estimates. For this purpose, we use the present-day photometric distances to molecular clouds. To study their kinematics, we use the radial velocities, trigonometric parallaxes, and proper motions of radio sources determined by various authors by VLBI.

\section{DATA}

In this paper, we use data on 230 molecular clouds from Schlafly et al. (2014). In the overwhelming majority of cases, these clouds are within $1 \mathrm{kpc}$ of the Sun. Schlafly et al. (2014) estimated the distances to them using photometric data on stars from the Pan-STARRS1 program (Kaiser et al. 2010) in combination with the distribution map of absorbing matter obtained as a result of the PLANCK space mission (Planck Collaboration 2011).

The following can be said in more detail about the photometric method by Schlafly et al. (2014). The distance to each cloud absorbing the light from the stars behind it was determined in two steps. In the first step, we determined the reddening and distance for each individual star in a given direction using Pan-STARRS1 photometry. In the second step, based on stars in the region selected for our analysis, we constructed the reddening function as a function of distance with the adoption of a simple dust screen model. The main idea of the method is to determine the region of discontinuity between the unreddened foreground stars and reddened background stars. The position of the discontinuity region gives an estimate of the distance modulus.

Up to 2000 stars from the region on the celestial sphere with a radius of $0.2^{\circ}$ were used 
to analyze each cloud. As a result, according to the compilers of the catalog, the mean random error in the distances to molecular clouds is less than $5 \%$, although the systematic uncertainties resulting from the quality of the applied stellar models are about $10 \%$. Thus, this is the biggest homogeneous modern catalog of accurate, directly measured distances to molecular clouds. The highly accurate estimates of the mean distances obtained by these authors for a number of nearby large molecular complexes, for example, in ScorpioCentaurus, Taurus, Perseus, and Orion, are of interest in studying the Gould Belt.

The radial velocities $V_{L S R}$ are known for quite a few nearby high-latitude clouds (Magnani et al. 1985). The random errors in the radial velocities do not exceed $5 \mathrm{~km} \mathrm{~s}^{-1}$, being, on average, $2 \mathrm{~km} \mathrm{~s}^{-1}$.

Young stars and protostars are closely associated with molecular clouds. These include, for example, Herbig-Haro objects or T Tauri stars. Many of them are observed in the radio band, in particular, by means of VLBI (Reid et al. 2014). At present, there are determinations of the radial velocities, trigonometric parallaxes, and proper motions for a small number of such objects from the solar neighborhood $0.6 \mathrm{kpc}$ in radius (Loinard 2013; Xu et al. 2013; Reid et al. 2014). This allows their total space velocities to be calculated with an error of about $10 \%$.

VLBI observations of five radio stars in Taurus: Hubble 4 and HDE 283572 (Torres et al. 2007), T Tau N ( $2 M_{\odot}$; Loinard et al. 2007), HP Tau/G2 (Torres et al. 2009), and V773 Tau (a spectroscopic binary with component masses of $1.55 \pm 0.11 M_{\odot}$ and $1.293 \pm 0.068 M_{\odot}$; Torres et al. 2012), were performed at $8.4 \mathrm{GHz}$ in continuum with the VLBA interferometer in the USA. All these stars are in the neighborhood of the molecular cloud Lynds 1495; they move parallel to one another with a small space velocity dispersion.

There are measurements in two clouds with maser sources, SVC13/NGC1333 (Hirota et al. 2008) and L 1448C (Hirota et al. 2011), in the region of the Perseus molecular complex. VLBI observations were performed at $22.2 \mathrm{GHz}$ within the Japanese VERA (VLBI Exploration of Radio Astrometry) program.

In the $\rho$ Ophiuchi molecular cloud, there are measurements for three stars at the edge close to the Sun: S1 $\left(\sim 6 M_{\odot}\right.$; Loinard et al. 2008), Dolidze-Arakelyan $21\left(\sim 2.2 M_{\odot}\right.$; Loinard et al. 2008), and YLW 15 (Rivera et al. 2015), as well as the maser source IRAS 16293-2422 at the far edge of the cloud (Imai et al. 2007). Note that YLW 15 is the only star in our list without a direct measurement of its trigonometric parallax.

There are two highly accurate results for the Orion molecular complex. The first is the VLBI measurement of the trigonometric parallax for the Orion Nebula (Menten et al. 2007 ) at $8.4 \mathrm{GHz}$ on the VLBA in the USA. This result was derived here as a mean of the observations of four different stars (GMR A, GMR F, GMR G, and GMR 12). The second highly accurate measurement was made for the BN/KL object at $43 \mathrm{GHz}$ ( $\mathrm{SiO}$ maser) within the VERA program (Kim et al. 2008). As we can see from Fig. 1 in Menten et al. (2007), the BN/KL object is located at the center of the previous sample of stars.

Finally, there are VLBI measurements of the trigonometric parallax and proper motion for the star EC 95 in Serpens (Dzib et al. 2010). This is a binary system consisting of a Herbig-Haro protostar with a mass of $4-5 M_{\odot}$ and a less massive T Tauri protostar. The systemic radial velocity of this binary has not yet been determined. Following Honma et al. (2012), we use $V_{L S R}=9 \pm 3 \mathrm{~km} \mathrm{~s}^{-1}$ taken from the velocity map of molecular clouds (Dame et al. 2001). 


\section{METHODS}

\section{Orientation Parameters}

We use the well-known method of determining the symmetry plane of a stellar system with respect to the main, in our case Galactic, coordinate system. The basics of this approach were outlined by Polak (1935); its description can be found in the book by Trumpler and Weaver (1953). Parenago (1951) and Pavlovskaya (1971) described the technique for estimating the errors of the sought-for angles. Recently, this method has been used to determine the orientation parameters for the system of Cepheids in the Galaxy (Bobylev 2013) and OB stars belonging to the Gould Belt (Bobylev and Bajkova 2014a).

In the rectangular coordinate system centered on the Sun, the $x$ axis is directed toward the Galactic center, the $y$ axis is in the direction of Galactic rotation $\left(l=90^{\circ}, b=0^{\circ}\right)$, and the $z$ axis is directed toward the North Galactic Pole; then, $x=r \cos l \cos b, y=$ $r \sin l \cos b$, and $z=r \sin b$, where $r$ is the star's heliocentric distance. Let $m, n, k$ be the direction cosines of the pole of the sought-for great circle from the $x, y, z$ axes. The sought-for symmetry plane of the stellar system is then defined as the plane for which the sum of the squares of the heights, $h=m x+n y+k z$, is at a minimum:

$$
\sum h^{2}=\min
$$

The sum of the squares

$$
h^{2}=x^{2} m^{2}+y^{2} n^{2}+z^{2} k^{2}+2 y z n k+2 x z k m+2 x y m n
$$

can be designated as $2 P=\sum h^{2}$. As a result, the problem is reduced to searching for the minimum of the function $P$ :

$$
2 P=a m^{2}+b n^{2}+c k^{2}+2 f n k+2 e k m+2 d m n,
$$

where the second-order moments of the coordinates $a=[x x], b=[y y], c=[z z], f=[y z]$, $e=[x z], d=[x y]$, written via the Gauss brackets, are the components of a symmetric tensor:

$$
\left(\begin{array}{lll}
a & d & e \\
d & b & f \\
e & f & c
\end{array}\right)
$$

whose eigenvalues $\lambda_{1,2,3}$ are found from the solution of the secular equation

$$
\left|\begin{array}{ccc}
a-\lambda & d & e \\
d & b-\lambda & f \\
e & f & c-\lambda
\end{array}\right|=0
$$

while the directions of the principal axes, $L_{1,2,3}$ and $B_{1,2,3}$, are found from the relations

$$
\begin{gathered}
\tan L_{1,2,3}=\frac{e f-(c-\lambda) d}{(b-\lambda)(c-\lambda)-f^{2}}, \\
\tan B_{1,2,3}=\frac{(b-\lambda) e-d f}{f^{2}-(b-\lambda)(c-\lambda)} \cos L_{1,2,3} .
\end{gathered}
$$


The errors in $L_{1,2,3}$ and $B_{1,2,3}$ are estimated according to the following scheme:

$$
\begin{gathered}
\varepsilon\left(L_{2}\right)=\varepsilon\left(L_{3}\right)=\frac{\varepsilon(\overline{x y})}{a-b}, \\
\varepsilon\left(B_{2}\right)=\varepsilon(\varphi)=\frac{\varepsilon(\overline{x z})}{a-c}, \\
\varepsilon\left(B_{3}\right)=\varepsilon(\psi)=\frac{\varepsilon(\overline{y z})}{b-c}, \\
\varepsilon^{2}\left(L_{1}\right)=\frac{\varphi^{2} \cdot \varepsilon^{2}(\psi)+\psi^{2} \cdot \varepsilon^{2}(\varphi)}{\left(\varphi^{2}+\psi^{2}\right)^{2}}, \\
\varepsilon^{2}\left(B_{1}\right)=\frac{\sin ^{2} L_{1} \cdot \varepsilon^{2}(\psi)+\cos ^{2} L_{1} \cdot \varepsilon^{2}\left(L_{1}\right)}{\left(\sin ^{2} L_{1}+\psi^{2}\right)^{2}},
\end{gathered}
$$

where

$$
\varphi=\cot B_{1} \cdot \cos L_{1}, \quad \psi=\cot B_{1} \cdot \sin L_{1},
$$

The three quantities $\overline{x^{2} y^{2}}, \overline{x^{2} z^{2}}$ and $\overline{y^{2} z^{2}}$ should be calculated in advance. Then,

$$
\begin{aligned}
& \varepsilon^{2}(\overline{x y})=\left(\overline{x^{2} y^{2}}-d^{2}\right) / n, \\
& \varepsilon^{2}(\overline{x z})=\left(\overline{x^{2} z^{2}}-e^{2}\right) / n, \\
& \varepsilon^{2}(\overline{y z})=\left(\overline{y^{2} z^{2}}-f^{2}\right) / n,
\end{aligned}
$$

where $n$ - is the number of stars. Thus, the algorithm for solving the problem consists in (i) setting up the function $2 P(3)$, (ii) seeking for the roots of the secular equation (5), and (iii) estimating the directions of the principal axes of the position ellipsoid from Eqs. (6)(16). As can be seen from Eqs. (12), the errors in the directions $L_{2}$ and $L_{3}$ coincide, while the errors in all the remaining directions are calculated independently of one another.

\section{Epicyclic Orbits}

Based on the epicyclic approximation (Lindblad 1927), it is easy to construct the orbits of objects in a coordinate system rotating around the Galactic center:

$$
\begin{aligned}
& x(t)=x_{0}+\frac{u_{0}}{\kappa} \sin (\kappa t)+\frac{v_{0}}{2 B}(1-\cos (\kappa t)), \\
& y(t)=y_{0}+2 A\left(x_{0}+\frac{v_{0}}{2 B}\right) t-\frac{\Omega_{0} v_{0}}{B \kappa} \sin (\kappa t)+\frac{2 \Omega_{0} u_{0}}{\kappa^{2}}(1-\cos (\kappa t)), \\
& z(t)=\frac{w_{0}}{\nu} \sin (\nu t)+z_{0} \cos (\nu t),
\end{aligned}
$$

where $t$ is the time in Myr (we take into account the relation pc/Myr $=0.978 \mathrm{~km} \mathrm{~s}^{-1}$ ); $A$ and $B$ are the Oort constants; $\kappa=\sqrt{-4 \Omega_{0} B}$ is the epicyclic frequency; $\Omega_{0}$ is the angular velocity of Galactic rotation of the local standard of rest; $\Omega_{0}=A-B ; \nu=\sqrt{4 \pi G \rho_{0}}$ is the frequency of vertical oscillations, where $G$ is the gravitational constant and $\rho_{0}$ is the 
stellar density in the solar neighborhood. The object's space velocity components $u, v, w$ are directed along the $x, y, z$ axes, respectively. The parameters $x_{0}, y_{0}, z_{0}$ and $u_{0}, v_{0}, w_{0}$ in the system of equations (17) denote the initial positions and velocities of the objects. The velocities $u, v, w$ are corrected for the peculiar motion of the Sun relative to the local standard of rest with components $\left(U_{\odot}, V_{\odot}, W_{\odot}\right)_{L S R}=(11.1,12.2,7.3) \mathrm{km} \mathrm{s}^{-1}$ (Schönrich et al. 2010). The local density of matter was taken to be $\rho_{0}=0.1 M_{\odot} \mathrm{pc}^{-3}$, as estimated by Holmberg and Flynn (2004), which gives $\nu=74 \mathrm{~km} \mathrm{~s}^{-1} \mathrm{kpc}^{-1}$. We used the Oort constants found from masers with measured trigonometric parallaxes, $A=16.9 \pm 1.2 \mathrm{~km}$ $\mathrm{s}^{-1} \mathrm{kpc}^{-1}$ and $B=-13.5 \pm 1.4 \mathrm{~km} \mathrm{~s}^{-1} \mathrm{kpc}^{-1}$ (Stepanishchev and Bobylev 2011), to which $\kappa=41 \mathrm{~km} \mathrm{~s}^{-1} \mathrm{kpc}^{-1}$ corresponds. The Sun's elevation above the Galactic plane is taken to be $z_{0}=16 \pm 2$ pc (Bobylev and Bajkova 2016).

\section{RESULTS AND DISCUSSION}

\section{Clouds}

The list by Schlafly et al. (2014) contains data for 230 molecular clouds. We selected 202 clouds located within $1 \mathrm{kpc}$ of the Sun. Based on this sample, we found the following orientation parameters:

$$
\begin{aligned}
& L_{1}=10 \pm 15^{\circ}, \quad B_{1}=10 \pm 15^{\circ}, \\
& L_{2}=103 \pm 1^{\circ}, \quad B_{2}=15 \pm 1^{\circ}, \\
& L_{3}=247 \pm 1^{\circ}, \quad B_{3}=73 \pm 2^{\circ} .
\end{aligned}
$$

The principal semi-axes of the ellipsoid in our method are determined to within a constant; their ratios are $\lambda_{1}: \lambda_{2}: \lambda_{3}=1: 0.67: 0.40$. If the size of the first semi-axis is taken to be $350 \mathrm{pc}$, then the sizes of the ellipsoid will be $350 \times 235 \times 140 \mathrm{pc}$, similar to those of the Lindblad ring. For example, when modelling the evolution of the neutral hydrogen clouds from which the Gould Belt was formed, Olano (1982) estimated the size of the system to be $364 \times 211$ pc. The coordinates of the ellipsoid center

$$
\begin{aligned}
& x_{0}=-118 \pm 15 \mathrm{pc} \\
& y_{0}=+54 \pm 11 \mathrm{pc} \\
& z_{0}=-12 \pm 8 \mathrm{pc}
\end{aligned}
$$

show the geometric center of the molecular cloud system to be located in the second quadrant.

The most important geometric characteristics of the Gould Belt found by various authors are given in Table 1 . These include the inclination of the system's symmetry plane to the Galactic plane $i$, the longitude of the ascending node $l_{\Omega}$, and the rectangular coordinates of the system's geometric center $x_{0}, y_{0}, z_{0}$. Stothers and Frogel (1974) studied the distribution of O-B5 stars. Olano (1982) found the coordinates of the center of the neutral hydrogen cloud by modelling the consequences of a supernova explosion. Westin (1985) considered a sample of nearby stars younger than 60 Myr. Torra et al. (2000) determined the geometric characteristics of the Gould Belt by analyzing the distribution of OB stars with their trigonometric parallaxes from the Hipparcos (1997) catalogue. Bobylev (2004) determined the geometric characteristics of the Gould Belt by analyzing 

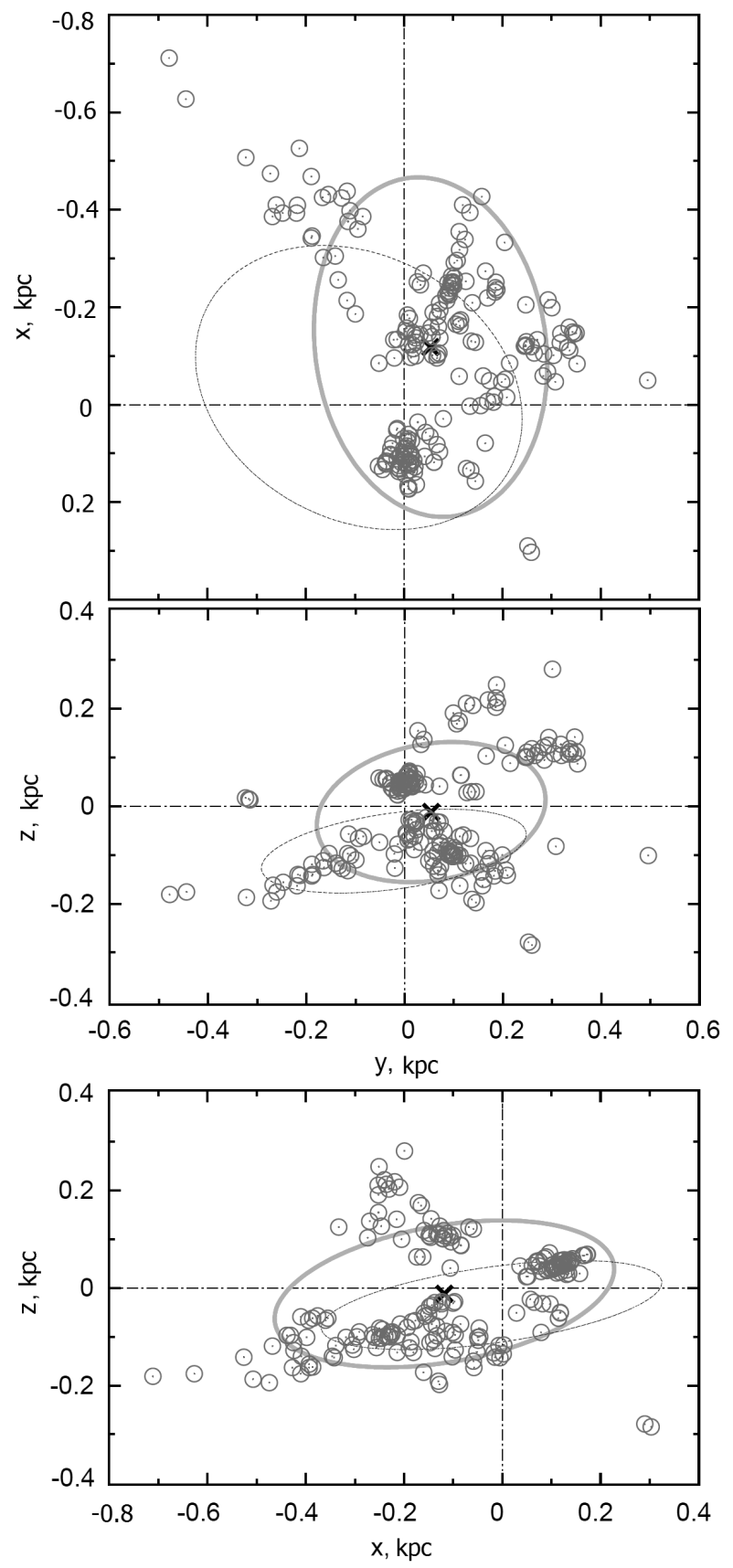

Figure 1: Positions of 202 molecular clouds in projection onto the Galactic $x y, y z$, and $x z$ planes. The Sun is at the coordinate origin. The thick line indicates the projections of the ellipsoid (18); the cross marks its center (19). The dashed line indicates the projections of the ellipsoid found from OB stars (Bobylev and Bajkova 2014a). 


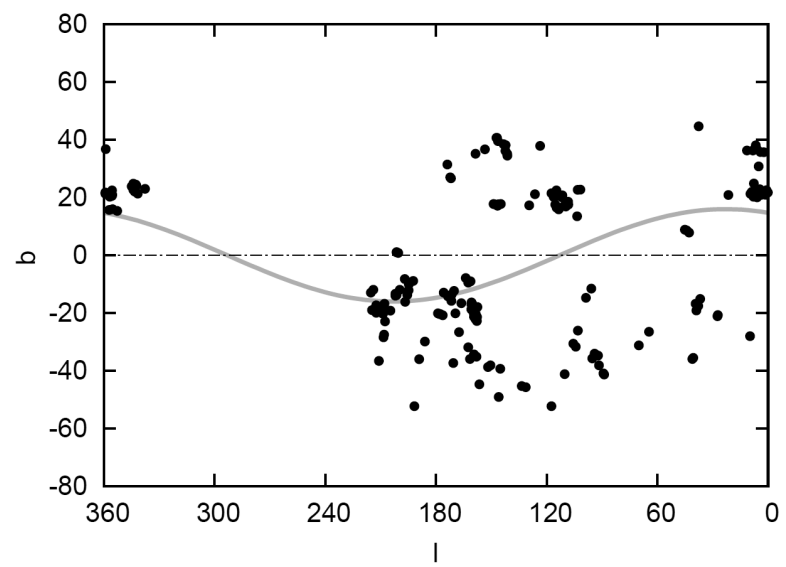

Figure 2: Distribution of 202 molecular clouds on the celestial sphere. The sine wave marks the great circle corresponding to the parameters (18).

Table 1: Geometric characteristics of the Gould Belt found by various authors

\begin{tabular}{|l|l|l|l|}
\hline Reference & $i$ & $l_{\Omega}$ & $\left(x_{0}, y_{0}, z_{0}\right) \pm\left(e_{x}, e_{y}, e_{z}\right), \mathrm{pc}$ \\
\hline Stothers and Frogel (1974) & $18 \pm 1^{\circ}$ & $295 \pm 2^{\circ}$ & - \\
Olano (1982) & - & - & $(-109,126)$ \\
Westin (1985) & $19^{\circ}$ & $270^{\circ}$ & - \\
Torra et al. (2000) & $16-22^{\circ}$ & $275-295^{\circ}$ & - \\
Perrot and Grenier (2003) & $17.2 \pm 0.5^{\circ}$ & $296 \pm 2^{\circ}$ & $(-104,0) \pm(4,4)$ \\
Bobylev (2004) & $17^{\circ}$ & $270^{\circ}$ & $(-141,51)$ \\
Piskunov et al. (2006) & - & - & $(-78,-53,-46)$ \\
Bobylev and Bajkova (2014a) & $13 \pm 1^{\circ}$ & $306 \pm 4^{\circ}$ & $(-35,-92,-22) \pm(13,14,5)$ \\
This paper & $17 \pm 2^{\circ}$ & $337 \pm 1^{\circ}$ & $(-118,54,-12) \pm(15,11,8)$ \\
\hline
\end{tabular}

the kinematics of $\mathrm{OB}$ stars. A sample of $\mathrm{OB}$ stars with errors in their trigonometric parallaxes less than 10\% was also considered by Bobylev and Bajkova (2014a), who, apart from the parameters listed in Table 1, also estimated the system's sizes, $350 \times 272 \times 78 \mathrm{pc}$ (this ellipsoid is shown in Fig. 1). Perrot and Grenier (2003) studied the distribution and evolution of molecular clouds; they estimated the sizes of the system to be $373 \times 233 \mathrm{pc}$. Piskunov et al. (2006) analyzed a sample of open star clusters, with this sample having been complete within 800 pc of the Sun.

The distributions of molecular clouds in projection onto the Galactic $x y, y z, x z$ planes are shown in Fig. 1. A significant difference along the $y$ axis in the position of the ellipse center found by Bobylev and Bajkova (2014a) from OB stars can be seen in Fig. 1: $x_{0}=-35 \pm 13 \mathrm{pc}, y_{0}=-92 \pm 14 \mathrm{pc}$, and $z_{0}=-22 \pm 5 \mathrm{pc}$. The situation with the geometric center of the Gould Belt calculated from the coordinates of open star clusters by Piskunov et al. (2006) is similar: $x_{0}=-78 \mathrm{pc}, y_{0}=-53 \mathrm{pc}$, and $z_{0}=-46 \mathrm{pc}$. As can be seen from Table 1, the coordinates (19) are fairly close to those found from gas clouds by Perrot and Grenier (2003). 


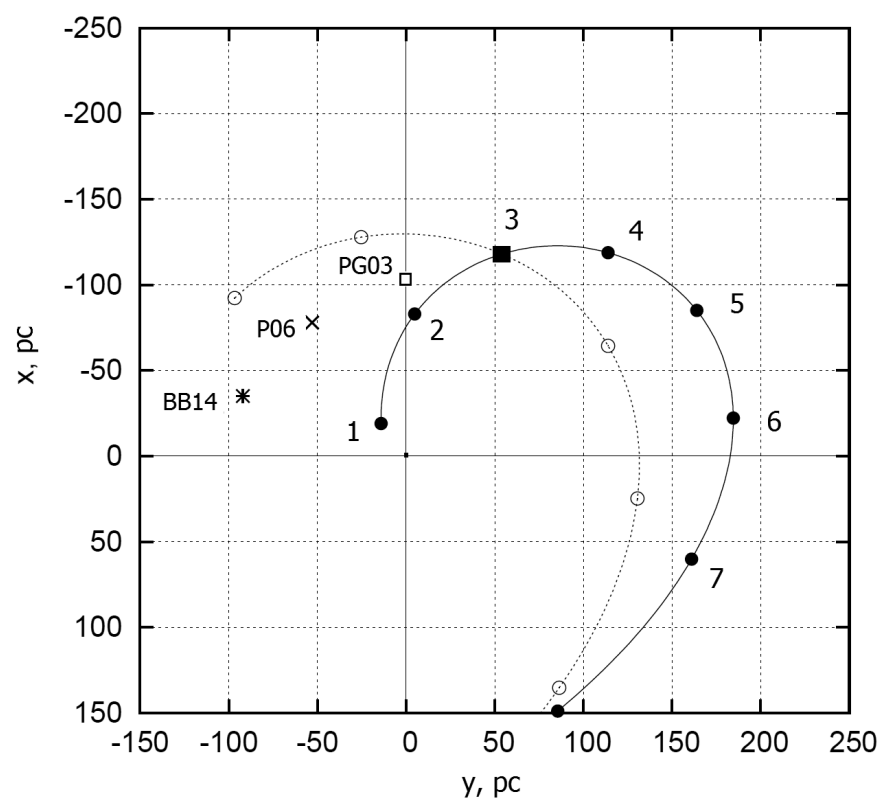

Figure 3: Trajectory of the center of the molecular cloud system relative to the local standard of rest (solid line). The filled circles are placed at intervals of $10 \mathrm{Myr}$. The filled square (point no. 3) marks the present-day center of this system. The second trajectory (the dotted line with open circles) was constructed with a different peculiar velocity of the Sun (for details, see the text). P06, BB14, and PG03 designate Piskunov et al. (2006), Bobylev and Bajkova (2014a), and Perrot and Grenier (2003), respectively.

Based on the parameters (18), we can calculate two more important characteristics: the inclination of the symmetry plane of the molecular cloud system to the Galactic plane $i=17 \pm 2^{\circ}$ and the longitude of the ascending node $l_{\Omega}=L_{3}+90^{\circ}=337 \pm 1^{\circ}$.

Note that when compiling their list, Schlafly et al. (2014) specially relied on highlatitude clouds. This was done, because it was extremely difficult to separate the Gould Belt clouds at low latitudes from the Galactic background clouds. In addition, there are virtually no data in the interval of longitudes $230^{\circ}<l<330^{\circ}$ in the list by Schlafly et al. (2014). Nevertheless, orientation parameters close to those of the Gould Belt are reliably obtained from 202 clouds. The distribution of these clouds on the celestial sphere is shown in Fig. 2, where the sine wave was constructed with $i=17^{\circ}$ and $l_{\Omega}=337^{\circ}$ corresponding to the solution (18). Since the clouds are high-latitude ones, far from the symmetry plane of the system (the Gould Belt), the size of the third axis of the ellipsoid (18) is approximately twice that of the Gould Belt found by various authors from stars, OB associations, and open clusters.

The discrepancy between the coordinates of the Gould Belt center found by various authors from open clusters and from the system of molecular clouds arouses interest. Is this scatter of results random? Is a relationship to the age and the individual pattern of evolution of the samples (star clusters and gas clouds) possible here?

First, it is necessary to understand how much we can be in error when calculating the coordinates of the center. It can be assumed that the coordinates of the system's 
Table 2: Velocities of molecular complexes calculated from radio stars and maser sources

\begin{tabular}{|l|c|c|c|}
\hline Complex & $n$ & $(U, V, W) \pm\left(e_{U}, e_{V}, e_{W}\right), \mathrm{km} \mathrm{s}^{-1}$ & $(x, y, z) \pm\left(e_{x}, e_{y}, e_{z}\right), \mathrm{pc}$ \\
\hline TAURUS & 5 & $(-16.7,-12.4,-9.2) \pm(1.0,0.2,0.3)$ & $(-132,19,-40) \pm(1,0,0)$ \\
ORION & 5 & $(-16.6,-19.6,1.8) \pm(3.8,2.5,1.7)$ & $(-343,-190,-137) \pm(4,2,2)$ \\
OPHIUCHUS & 3 & $(-7.0,-14.7,-7.3) \pm(2.1,0.6,1.0)$ & $(114,-14,34) \pm(6,1,2)$ \\
PERSEUS & 2 & $(-19.9,-20.7,-6.5) \pm(2.3,0.7,0.3)$ & $(-203,81,-83) \pm(15,6,6)$ \\
IRAS 16293-2422 & 1 & $(-7.9,-31.5,-6.0) \pm(4.4,6.0,2.4)$ & $(171,-18,49) \pm(34,4,9)$ \\
EC 95 & 1 & $(-3.8,-9.1,-5.3) \pm(2.7,1.7,0.3)$ & $(352,216,39) \pm(3,2,0)$ \\
\hline
\end{tabular}

center were shifted into the second quadrant due to the absence of data in the interval of longitudes $230^{\circ}<l<330^{\circ}$. However, it should be noted that there are no considerable number of molecular clouds belonging to the Gould Belt in the interval of longitudes $270^{\circ}<l<330^{\circ}$. This can be clearly seen from Fig. 7 in Dame et al. (1987), where the distribution of molecular complexes within $1 \mathrm{kpc}$ of the Sun with an indication of their masses and heights above the Galactic plane are shown. There is a complex of clouds in Vela only in a direction $l \approx 280^{\circ}$ at a distance of about 500 pc, but it is located in the Galactic plane $\left(|b|<25^{\circ}\right)$. To compensate for the absence of data on molecular clouds, Perrot and Grenier (2003) took the data on neutral hydrogen clouds in the region $270^{\circ}<l<330^{\circ}$, as indicated in Figs. 1 and 2 of these authors. Therefore, it is important that the parameters (19) are closest to the results of Perrot and Grenier (2003) obtained by analyzing a sample of gas clouds distributed quite uniformly in longitude.

Then, we decided to trace the motion of the Gould Belt center in a time interval comparable to its age (40-60 Myr). For this purpose, it is necessary to calculate the velocity of the molecular cloud system relative to the local standard of rest. We used 105 clouds with the estimates of their radial velocities $V_{L S R}$ from Magnani et al. (1985). We passed from the velocities $V_{L S R}$ to $V_{r}$ via the parameters of the standard solar motion $\left(U_{\odot}, V_{\odot}, W_{\odot}\right)_{L S R}=(10.3,15.3,7.7) \mathrm{km} \mathrm{s}^{-1}$. Then,

$$
V_{r}=-u_{0} \cos b \cos l-v_{0} \cos b \sin l-w_{0} \sin b+r A \cos ^{2} b \sin 2 l,
$$

where $A=0.5 R_{0} \Omega_{0}^{\prime}$ is the Oort constant and $\Omega_{0}^{\prime}$ is the first derivative of the angular velocity of Galactic rotation. Solving the system of conditional equations (20) by the least-squares method, we found the following group velocity components:

$$
\begin{aligned}
& u_{0}=10.6 \pm 0.9 \mathrm{~km} \mathrm{~s}^{-1}, \\
& v_{0}=18.2 \pm 1.7 \mathrm{~km} \mathrm{~s}^{-1}, \\
& w_{0}=6.8 \pm 1.5 \mathrm{~km} \mathrm{~s}^{-1},
\end{aligned}
$$

The Oort constant was $A=13.2 \pm 6.4 \mathrm{~km} \mathrm{~s}^{-1} \mathrm{kpc}^{-1}$, and the error per unit weight was $\sigma_{0}=4.9 \mathrm{~km} \mathrm{~s}^{-1}$. The velocities $(21)$ are in good agreement with the velocity components of the Gould Belt $\left(u_{0}, v_{0}, w_{0}\right)=(9.26,15.93,6.96) \pm(0.72,0.72,0.60) \mathrm{km} \mathrm{s}^{-1}$ calculated by Bobylev (2006) using 49 open star clusters. Only the motion along the y coordinate differs by $\Delta v_{0} \sim 2 \mathrm{~km} \mathrm{~s}^{-1}$. Note that the Sun's peculiar motion relative to the local standard of 


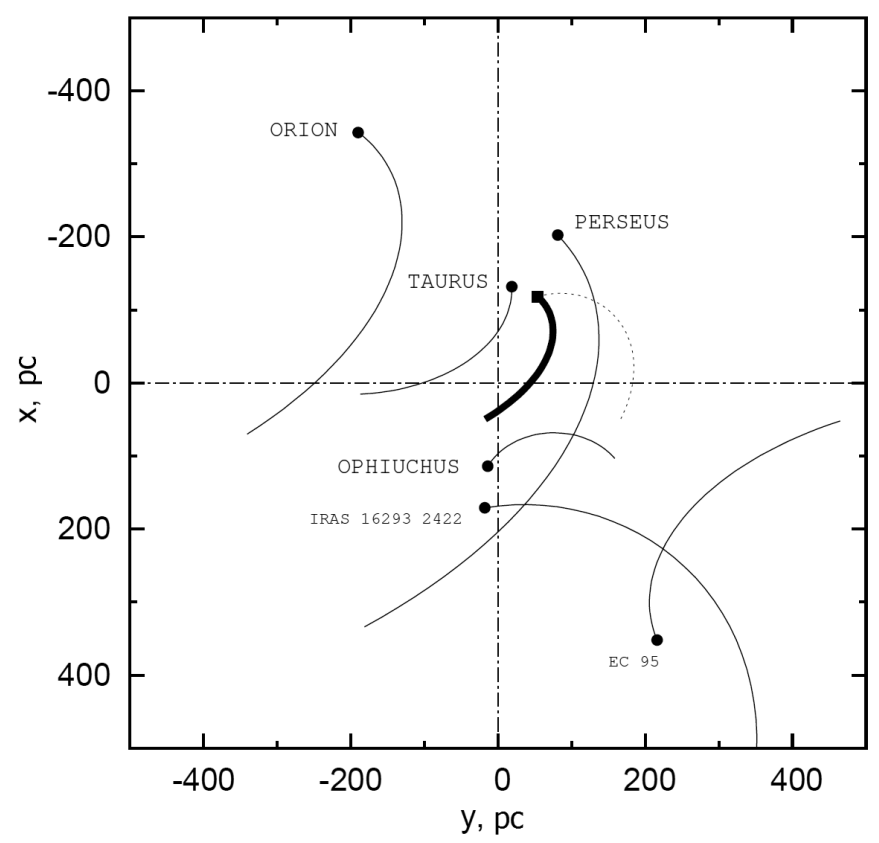

Figure 4: Trajectories of four molecular complexes and two stars relative to the local standard of rest in the past in an interval of 40 Myr. The trajectory of the center of this system is highlighted by the thick line.

rest $\left(U_{\odot}, V_{\odot}, W_{\odot}\right)_{L S R}$ makes a major contribution to the group velocity (21). The residual velocity is quite low: it is virtually zero along the $z$ coordinate, while it is $4-10 \mathrm{~km} \mathrm{~s}^{-1}$ along each of the $x$ and $y$ coordinates, depending on the adopted solar velocity.

The trajectory of the Gould Belt center relative to the local standard of rest calculated on the basis of Eqs. (17) is presented in Fig. 3. We took the velocities (21) for its construction. These velocities were assigned to the determined center of molecular clouds (point no. 3 in Fig. 3). The segments on the trajectory are given at intervals of 10 Myr. Numbers 1-7 increase into the past, i.e., points 1 and 2 are the future ones for point 3.

The solid curve with filled circles in Fig. 3 was calculated with the velocities corrected for the Sun's peculiar motion relative to the local standard of rest with $\left(U_{\odot}, V_{\odot}, W_{\odot}\right)_{L S R}=$ $(11.1,12.2,7.3) \mathrm{km} \mathrm{s}^{-1}$ from Schönrich et al. (2010), while the dotted curve was calculated with the velocities corrected with $\left(U_{\odot}, V_{\odot}, W_{\odot}\right)_{L S R}=(6.0,10.6,6.5) \mathrm{km} \mathrm{s}^{-1}$ from Bobylev and Bajkova (2014b). As can be seen from the figure, the trajectory of the center of the molecular cloud system depends noticeably on the adopted initial parameters.

Four centers are indicated in Fig. 3: for the system of open star clusters (P06), the system of OB stars (BB14), the system of hydrogen clouds (PG03), and the system of molecular clouds (point no. 3). We get the impression that they are all fall nicely on some average trajectory of the Gould Belt. But why does the gas component lag behind the stellar one in this motion? It is impossible to completely explain this discrepancy by the random errors in the coordinates of the center. 


\section{Complexes of Clouds}

Table 2 provides the velocities of the molecular cloud complexes calculated from the VLBI measurements of radio stars and maser sources. Column 1 gives the name of the complex or maser source, column 2 gives the number of sources that we used to derive the mean motion, and column 3 and 4 give the mean velocities and mean coordinates, respectively. As a result, we have four complexes and two stars.

Figure 4 presents the trajectories of the molecular complexes relative to the local standard of rest in the past in an interval of $40 \mathrm{Myr}$. The trajectory of the center of this system highlighted by the thick line was calculated for their group velocity $\left(u_{0}, v_{0}, w_{0}\right)=(13.6,15.0,6.4) \pm(1.7,2.4,1.7) \mathrm{km} \mathrm{s}^{-1}$ ascribed to the center of the molecular cloud system (19) using the peculiar velocity of the Sun relative to the local standard of rest $\left(U_{\odot}, V_{\odot}, W_{\odot}\right)_{L S R}=(11.1,12.2,7.3) \mathrm{km} \mathrm{s}^{-1}$ from Schönrich et al. (2010). The dotted line in the figure indicates the trajectory that is the main trajectory of the center of the cloud system in the previous figure (Fig. 3).

As can be seen from the figure, the motions of the complexes in Orion, Taurus, and Perseus are coordinated: these trajectories are virtually parallel both to one another and to the trajectory of the center. We may conclude that the random velocity component is suppressed. In addition, the center indicated in the figure is fairly close to the geometric center of the sample even $40 \mathrm{Myr}$ ago. It can be seen from the figure that when each of the trajectories is directed into the future, an expansion of the entire structure of the Gould Belt from a point close to its center is observed.

Using VLBI measurements of radio stars, Rivera et al. (2015) performed a similar analysis of the kinematics of the complexes in Orion, Taurus, and Ophiuchus, though without constructing their trajectories. These authors found the velocities of these complexes to exhibit an expansion effect. When more such kinematic data will appear, it will be interesting to trace the spatial evolution of the system of molecular clouds in the Gould Belt in details.

\section{CONCLUSIONS}

Using data on nearby (located within $1 \mathrm{kpc}$ of the Sun) molecular clouds with highly accurate distance estimates from Schlafly et al. (2014), we redetermined the spatial orientation parameters of this system. In particular, the inclination of the symmetry plane of this system to the Galactic plane and the longitude of the ascending node were found to be $i=17 \pm 2^{\circ}$ and $l_{\Omega}=337 \pm 1^{\circ}$, respectively. These values show a very close association of the clouds with the Gould Belt, despite the gaps in the data, and that the overwhelming majority of clouds are high-latitude ones. The sizes of the approximation ellipsoid are $350 \times 235 \times 140 \mathrm{pc}$, where the third axis is a factor of 2-2.5 larger than the third axis in the ellipsoid of the Gould Belt found by various authors from young stars, OB associations, or open star clusters. We showed a great difference between the coordinates of the Gould Belt center determined by various authors from open star clusters, OB associations, and gas clouds.

Based on the radial velocities of a sample of 105 high-latitude clouds, we found their group velocity relative to the Sun $\left(u_{0}, v_{0}, w_{0}\right)=(10.6,18.2,6.8) \pm(0.9,1.7,1.5) \mathrm{km} \mathrm{s}^{-1}$. 
Using these values and based on the epicyclic approximation, we constructed the trajectory of the center of the system of molecular clouds in the Gould Belt in an interval of $\sim 60$ Myr.

Using highly accurate data on maser sources associated with low-mass $\left(1-2 M_{\odot}\right)$ protostars, we calculated the mean space velocities of four molecular complexes in Orion, Taurus, Perseus, and Ophiuchus. We showed the motion of these complexes in the past to be not random. Such data are still scarce, but invoking the GAIA measurements will allows us to make considerable progress in understanding the evolution of the molecular cloud system and the Gould Belt as a whole.

\section{ACKNOWLEDGMENTS}

We are grateful to the referees for their helpful remarks that contributed to an improvement of this paper. This work was supported by the "Transitional and Explosive Processes in Astrophysics" Program P-41 of the Presidium of Russian Academy of Sciences.

\section{REFERENCES}

1. P.A.R. Ade, N. Aghanim, M. Arnaud, M. Ashdown, J. Aumont, C. Baccigalupi, A. Balbi, A.J. Banday, et al. (Planck Collab.), Astron. Astrophys. 536, A19 (2011).

2. K. Bekki, Mon. Not. R. Astron. Soc. 398, L36 (2009).

3. A. Blaauw, Bull. Astron. Inst. Netherlands 11, 414 (1952).

4. V.V. Bobylev, Astron. Lett. 30, 159 (2004).

5. V. V. Bobylev, Astron. Lett. 32, 816 (2006).

6. V.V. Bobylev, Astron. Lett. 39, 753 (2013).

7. V.V. Bobylev and A.T. Bajkova, Astron. Lett. 40, 783 (2014).

8. V.V. Bobylev, Astrophysics 57, 583 (2014).

9. V.V. Bobylev, and A.T. Bajkova, Mon. Not. R. Astron. Soc. 441, 142 (2014b).

10. V.V. Bobylev and A.T. Bajkova, Astron. Lett. 42, 1 (2016).

11. F. Comerón and J. Torra, Astron. Astrophys. 261, 94 (1992).

12. F. Comerón and J. Torra, Astron. Astrophys. 281, 354 (1994).

13. T.M. Dame, H. Ungerechts, R.S. Cohen, E.J. de Geus, I.A. Grenier, J. May, D.C. Murphy, L.-A. Nyman, and P. Thaddeus, Astrophys. J. 322, 706 (1987).

14. T.M. Dame, D. Hartmann, and P. Thaddeus, Astrophys. J. 547, 792 (2001).

15. S. Dzib, L. Loinard, A.J. Mioduszewski, A.F. Boden, L.F. Rodríguez, and R.M. Torres,Astrophys. J. 718, 610 (2010).

16. S. Dzib, L. Loinard, L.F. Rodríguez, A.J. Mioduszewski, G.N. Ortiz-Leon, M.A. Kounkel, G. Pech, J.L. Rivera, et al., Astrophys. J. 801, 91 (2015).

17. Yu.N. Efremov, Sites of Star Formation in Galaxies (Nauka, Moscow, 1989) [in Russian].

18. J.A. Frogel and R. Stothers, Astron. J. 82, 890 (1977).

19. The Hipparcos and Tycho Catalogues, ESA SP-1200 (1997).

20. T. Hirota, T. Bushimata, Y.K. Choi, M. Honma, H. Imai, I. Hiroshi, K. Iwadate, T. Jike, et al., Publ. Astron. Soc. Jpn. 60, 37 (2008).

21. T. Hirota, M. Honma, H. Imai, K. Sunada, Y. Ueno, H. Kobayashi, and N. Kawaguchi, Publ. Astron. Soc. Jpn. 63, 1 (2011).

22. J. Holmberg and C. Flynn, Mon. Not. R. Astron. Soc. 352, 440 (2004).

23. M. Honma, T. Nagayama, K. Ando, T. Bushimata, Y.K. Choi, T. Handa, T. Hirota, H. Imai, et al., Publ. Astron. Soc. Jpn. 64, 136 (2012). 
24. H. Imai, K. Nakashima, T. Bushimata, Y.K. Choi, T. Hirota, M. Honma, K. Horiai, N. Inomata, et al., Publ. Astron. Soc. Jpn. 59, 1107 (2007).

25. M.K. Kim, T. Hirota, M. Honma, H. Kobayashi, T. Bushimata, Y.K. Choi, H. Imai, K. Iwadate, et al., Publ. Astron. Soc. Jpn. 60, 991 (2008).

26. V. Konyves, Ph. Andre, A. Men'shchikov, P. Palmeirim, D. Arzoumanian, N. Schneider, A. Roy, P. Didelon, et al., Astron. Astrophys. 584, 91 (2015).

27. C.J. Lada, in Young Stars and Planets Near the Sun, Proceedings of the IAU Symposium No. 314, Ed. by J.H. Kastner, B. Stelzer, and S.A. Metchev (2015).

28. J.R.D. Lepiné and G. Duvert, Astron. Astrophys. 286, 60 (1994).

29. B. Lindblad, Ark. Mat. Astron. Fys. A 20 (17) (1927).

30. P.O. Lindblad, Bull. Astron. Inst. Netherland 19, 34 (1967).

31. P.O. Lindblad, Astron. Astrophys. 363, 154 (2000).

32. L. Loinard, R.M. Torres, A.J. Mioduszewski, L.F. Rodriguez, R.A. Gonzalez-Lopezlira, R. Lachaume, V. Vazquez, and E. Gonzalez, Astrophys. J. 671, 546 (2007).

33. L. Loinard, R.M. Torres, A.J. Mioduszewski, and L.F. Rodriguez, Astrophys. J. 675, L29 (2008).

34. L. Loinard, in Proceedings of the IAU Symposium No. 289 on Advancing the Physics of Cosmic Distances, Beijing, China, Aug. 27-31, 2012, Ed. by R. de Grijs and G. Bono (2013).

35. L. Magnani, L. Blitz, and L. Mundy, Astrophys. J. 295, 402 (1985).

36. K.M. Menten, M.J. Reid, J. Forbrich, and A. Brunthaler, Astron. Astrophys. 474, 515 (2007).

37. E. Moreno, E.J. Alfaro, and J. Franco, Astrophys. J. 522, 276 (1999).

38. C.A. Olano, Astron. Astrophys. 112, 195 (1982).

39. C.A. Olano, Astron. Astrophys. 121, 295 (2001).

40. F. Palla and S. W. Staler, Astrophys. J. 540, 255 (2000).

41. J. Palouš, Astrophys. Space Sci. 276, 359 (2001).

42. J. Palouš, and S. Ehlerová, arXiv:1406.6248 (2014).

43. P.P. Parenago, Tr. GAISh 20, 26 (1951).

44. E.D. Pavlovskaya, in Practical Works on Stellar Astronomy, Ed. by P.G. Kulikovskii (Nauka, Moscow, 1971), p. 162 [in Russian].

45. C.A. Perrot and I.A. Grenier, Astron. Astrophys. 404, 519 (2003).

46. A.E. Piskunov, N.V. Kharchenko, S. Röser, E. Schilbach, and R.-D. Scholz, Astron. Astrophys. 445, 545 (2006).

47. I.F. Polak, Introduction to Stellar Astronomy (ONTI, Moscow, Leningrad, 1935) [in Russian].

48. W.G.L. Pöppel, Fundam. Cosm. Phys. 18, 1 (1997).

49. W.G.L. Pöppel, ASP Conf. Ser. 243, 667 (2001).

50. W.G.L. Pöppel and P. Marronetti, Astron. Astrophys. 358, 299 (2000).

51. T. Preibish and H. Zinnecker, Astron. J. 117, 2381 (1999).

52. M.J. Reid, K.M. Menten, A. Brunthaler, X.W. Zheng, T.M. Dame, Y. Xu, Y. Wu, B. Zhang, et al., Astrophys. J. 783, 130 (2014).

53. J.L. Rivera, L. Loinard, S.A. Dzib, G.N. Ortiz-Leon, L.F. Rodriguez, and R.M. Torres, Astrophys. J. 807, 119 (2015).

54. E.F. Schlafly, G. Green, D.P. Finkbeiner, H.- W. Rix, E.F. Bell, W.S. Burgett, K.C. Chambers, P.W. Draper, et al., Astrophys. J. 786, 29 (2014).

55. R. Schönrich, J. Binney, and W. Dehnen, Mon. Not. R. Astron. Soc. 403, 1829 (2010).

56. A.S. Stepanishchev and V.V. Bobylev, Astron. Lett. 37, 254 (2011).

57. R. Stothers and J.A. Frogel, Astron. J. 79, 456 (1974). 
58. J. Torra, D. Fernańdez, and F. Figueras, Astron. Astrophys. 359, 82 (2000).

59. R.M. Torres, L. Loinard, A.J. Mioduszewski, and L.F. Rodriguez, Astrophys. J. 671, 1813 (2007).

60. R.M. Torres, L. Loinard, A.J. Mioduszewski, and L.F. Rodriguez, Astrophys. J. 698, $242(2009)$.

61. R.M. Torres, L. Loinard, A.J. Mioduszewski, A.F. Boden, R. Franco-Hernandez, W.H.T. Vlemmings, and L.F. Rodriguez, Astrophys. J. 747, 18 (2012).

62. R.J. Trumpler and H.F. Weaver, Statistical Astronomy (Univ. California Press, Berkely, 1953).

63. T.N.G. Westin, Astron. Astrophys. Suppl. Ser. 60, 99 (1985).

64. Y. Xu, J.J. Li, M.J. Reid, K.M. Menten, X.W. Zheng, A. Brunthaler, L. Moscadelli, T.M. Dame, and B. Zhang, Astrophys. J. 769, 15 (2013).

65. P.T. de Zeeuw, R. Hoogerwerf, J.H.J. de Bruijne, A.G.A. Brown, and A. Blaauw, Astron. J. 117, 354 (1999). 